\title{
CO-WELL-POWERED REFLECTIVE SUBCATEGORIES
}

\author{
RUDOLF-E. HOFFMANN
}

\begin{abstract}
A full isomorphism-closed subcategory $A$ of a complete wellpowered and co-well-powered category $C$ is both co-well-powered (in its own right) and reflective in $C$ if and only if

(a) $A$ is closed in $C$ under the formation of ( $U$-small-indexed) limits, and

(b) the epi-reflective hull $B$ of $A$ in $C$ is co-well-powered.
\end{abstract}

A full isomorphism-closed subcategory $\mathcal{Y}$ of a well-powered and co-well-powered complete category $X$ is epi-reflective in $X$ (i.e. full, isomorphism-closed and reflective with epimorphic reflection morphisms) if and only if $\mathcal{Y}$ is stable in $X$ under the formation of products (indexed over $U$-small sets) and under extremal subobjectswhere $U$ denotes a fixed universe (cf. $[2$, p. 87; 6, p. 1276; 7, p. 356]). Indeed, a slightly weaker requirement suffices to ensure the epi-reflectiveness of $y$ in $x: y$ is closed under ( $U$-small-indexed) products and strongly closed under difference kernels, i.e. whenever

$$
A \underset{f}{\rightarrow} \underset{h}{\stackrel{g}{\rightrightarrows}} C
$$

is a difference kernel (or equalizer) in $\mathcal{X}$ with $B \in \mathrm{Ob} \mathcal{Y}$, then $A \in \mathrm{Ob} \mathcal{Y}[3,10.2 .1]$.

The smallest epi-reflective subcategory $D$ of a well-powered and co-well-powered complete category $C$ containing a given subcategory $\mathcal{X}$ of $C$, the "epi-reflective hull" $D$ of $X$ in $C$, consists of all $C$-objects which are (domains of ) extremal subobjects of products (over a $U$-small index set) of members of $\mathrm{Ob} X$. Every full isomorphism-closed reflective subcategory $A$ of a well-powered and co-well-powered complete category $C$ is both mono-reflective and (consequently) epi-reflective in the epi-reflective hull $B$ of $A$ in $C$ [1]. Sharpening results in [1 and 7], it is observed in [4] that a full subcategory $A$ of a complete, well-powered and co-well-powered category $C$ is reflective in $C$ if (i) and (ii) are satisfied:

(i) $A$ is stable in $C$ under the formation of ( $U$-small-indexed) limits;

(ii) the epi-reflective hull $B$ of $A$ in $C$ is co-well-powered.

(Indeed, a difference kernel

$$
X \stackrel{u}{\rightarrow} Y \underset{w}{\stackrel{v}{\rightrightarrows}} Z
$$

in $B$ with $Y \in \mathrm{Ob} A$ yields a difference kernel

$$
X \stackrel{u}{\rightarrow} Y \underset{m v}{\stackrel{m v}{\rightrightarrows}} A
$$

for some extremal monomorphism $m: Z \rightarrow A$ in $C$ with $A \in \mathrm{Ob} A$; hence $X \in \mathrm{Ob} A$ by hypothesis. Consequently, $A$ is epi-reflective in $B$.)

While (i) is clearly necessary for reflectiveness of $A$ in $C$, condition (ii) is not. Here we wish to add the following observations.

Received by the editors December 13, 1982.

1980 Mathematics Subject Classification. Primary 18 A40.

Key words and phrases. Reflective subcategory, co-well-powered category, epi-reflective hull. 
1. A full subcategory $A$ of a complete, well-powered and co-well-powered category $C$ is co-well-powered if the epi-reflective hull $B$ of $A$ in $C$ is also.

Indeed, the inclusion $A \rightarrow B$ preserves epimorphisms $[5,2.2]$.

2. A full isomorphism-closed subcateogry $A$ of a complete well-powered and cowell-powered category $C$ is both co-well-powered (in its own right) and reflective in $C$ if and only if

(a) $A$ is closed in $C$ under the formation of ( $U$-small-indexed) limits, and

(b) the epi-reflective hull $B$ of $A$ in $C$ is co-well-powered.

Proof. It remains to establish the necessity of (b). For $X \in \mathrm{Ob} C$, let $r_{X}: X \rightarrow$ $R(X)$ denote the $A$-reflection morphism of $X$. Now let $B \in \mathrm{Ob} B$. For every $B$-epimorphism $e: B \rightarrow Y$ we obtain a commutative square:

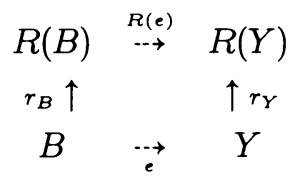

Since $r_{Y}$ is a $B$-epimorphism, so is $r_{Y} e=R(e) r_{B}$; hence so is $R(e)$. Consequently, $R(e)$ is an epimorphism in $A$. There are suitable isomorphisms $j_{e}$ in $A$ with domain $R(Y)$ so that we obtain a mapping $\varphi: e \mapsto j_{e} R(e)$ from a representative system of $B$ epimorphisms with domain $B$ into a representative system of $A$-epimorphisms with domain $R(B)$. The latter set is $U$-small by hypothesis. Since $r_{Y}$ is an (extremal) monomorphism and since $C$ is well-powered, the fibers of this mapping $\varphi$ (i.e. the inverse images of single elements) are $U$-small. As a consequence, the domain of $\varphi$ is also $U$-small, i.e. $B$ is co-well-powered.

Necessary and sufficient conditions for a subcategory to be co-well-powered reflective are also given in [1, Theorem 3].

Note ADDED IN PROOF. A careful examination of the proofs of the results leading to the theorem obtained above shows that the latter can be extended to a complete category $C$ with a "well-founded" bicategory structure $(E, M)[7$, p. 355] when the epi-reflective hull $B$ of $A$ is replaced by the $E$-reflective hull of $A$ in $C$. (This transfers co-well-poweredness from compact $T_{2}$-spaces to completely Hausdorff spaces (with continuous maps) as well as to every intermediate full subcategory.)

\section{REFERENCES}

1. S. Baron, Reflectors as compositions of epi-reflectors, Trans. Amer. Math. Soc. 136 (1969), 499-508.

2. P. Freyd, Abelian categories, Harper \& Row, New York, 1964.

3. H. Herrlich, Topologische Reflexionen und Coreflexionen, Lecture Notes in Math., vol. 78, Springer-Verlag, Berlin 1968.

4. __ Epireflective subcategories of TOP need not be cowellpowered, Comment. Math. Univ. Carolin. 16 (1975), 713-716.

5. R.-E. Hoffmann, Factorization of cones II, with an application to weak Hausdorff spaces (Proc. Conf. Categorical Aspects of Topology and Analysis, Carleton Univ., Ottawa, 1980), Lecture Notes in Math., vol. 915, Springer-Verlag, Berlin, 1982, pp. 148-170.

6. J. R. Isbell, Natural sums and abelianizing, Pacific J. Math. 14 (1964), 1265-1281.

7. J. F. Kennison, Full reflective subcategories and generalized covering spaces, Illinois J. Math. 12 (1968), 353-365.

Fachbereich Mathematik, Universität Bremen, D-2800 Bremen, Federal Republic OF GERMANY 